\title{
Análise dos Valores SUS para a Revascularização Miocárdica Percutânea Completa em Multiarteriais
}

\author{
Analysis of Brazilian Public Health System Values for Complete Percutaneous Myocardial Revascularization in \\ Multi-Arterial Patients \\ George César Ximenes Meireles ${ }^{1,2}$, Marcos Kiyoshi Sumita ${ }^{1}$, Antonio Artur da Cruz Forte ${ }^{1}$, Desidério Favarato ${ }^{3}$, \\ Rodrigo Costa Quintão ${ }^{1}$, Luciano Maurício de Abreu Filho ${ }^{1}$ \\ Hospital Stela Maris"; Hospital do Servidor Público Estadual "Francisco Morato de Oliveira"2, Instituto do Coração da FMUSP3, São Paulo, SP - Brasil
}

\section{Resumo}

Fundamento: O Sistema Único de Saúde (SUS) estabelece que a angioplastia coronariana com o implante de duplo stent não deve exceder $\mathbf{2 0} \%$ das angioplastias, resultando na necessidade de escalonar a maioria dos procedimentos nos pacientes com doença multiarterial.

Objetivo: O objetivo do presente estudo foi avaliar os valores remunerados pelo SUS para a obtenção da revascularização miocárdica percutânea completa em pacientes do SUS com doença multiarterial relacionados ao número de procedimentos necessários e de stents implantados.

Métodos: Foram incluídos 141 pacientes com doença coronariana multiarterial, submetidos à revascularização completa com sucesso pelo implante de stent, com coronariografia aos 6 meses pós-implante. A revascularização completa foi definida como o tratamento percutâneo de todas as lesões com percentual de estenose $>70 \%$, em vasos com diâmetro $>2 \mathrm{~mm}$. Para análise dos custos, foram considerados os valores da Tabela SIH/SUS de R\$ 2.263,77 para o procedimento e R\$ 2.034,23 por stent implantado.

Resultados: No período de 07/2006 a 12/2007 foram implantados 416 stents em 141 pacientes. A idade média foi de 59,7 \pm 9,9 anos, com predomínio do sexo masculino (68,1\%). O número de vasos foi 356 e o número de lesões 416 . Para a obtenção da revascularização completa pelo implante de stent coronáriano foi necessário o escalonamento em até 4 procedimentos. O tempo

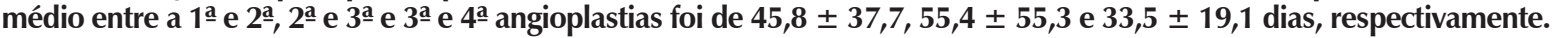

Conclusão: A revascularização percutânea completa em pacientes do SUS com doença coronariana multiarterial, realizada em sua grande maioria de forma escalonada, ocasiona considerável elevação de gastos públicos devido ao aumento do número de procedimentos. (Arq Bras Cardiol. 2010; [online]. ahead print, PP.0-0)

Palavras-chave: Doença da artéria coronariana, stents, revascularização miocárdica, política de saúde.

\section{Abstract}

Background: The Brazilian Public Health System (SUS, acronym in Portuguese) establishes that coronary angioplasty with the double implant stent must not exceed $20 \%$ of the angioplasties, resulting in the need of assigning most of the procedures in patients with multiarterial disease.

Objective: The objective of the present study was to assess the paid values by the SUS in order to obtain the complete percutaneous myocardial revascularization in the SUS patients with the multiarterial disease, related to the number of necessary procedures and of implanted stents.

Methods: A total of 141 patients with multiarterial coronary disease, submitted to a successful complete revascularization, were included by the stent implant with coronariography in a 6-month period of post-implant. The complete revascularization was defined as the percutaneous treatment of all stenosis $>70 \%$ in vessels with diameter $>2 \mathrm{~mm}$. For the costs analysis, the values from the Sistema de Informações Hospitalares $(S I H)$ of the SUS table were considered as $R \$ 2,263.77$, for the procedure; and $R \$ 2,034.23$, per implanted stent.

Results: In the period from 7/2006 to 12/2007, 416 stents were implanted in 141 patients. The mean age was of $59.7 \pm 9.9$ years old, prevailing the male sex (68.1\%). The number of vessels was 356 and the lesions number corresponded to 416 . In order to obtain the complete revascularization by the coronary stent implant, it was necessary to stagger in up to four procedures. The mean time between the $1^{\text {st }}$ and $2^{\text {nd }}, 2^{\text {nd }}$ and $3^{\text {rd }}$ and $3^{\text {rd }}$ and $4^{\text {th }}$ angioplasties was of $45.8 \pm 37.7 ; 55.4 \pm 55.3$ and $33.5 \pm 19.1$ days, respectively.

Conclusion: The complete percutaneous revascularization in patients from the SUS with the multiarterial coronary disease, carried out in most of them staggered, causes considerable elevation of public expenses due to the increase of procedures' number. (Arq Bras Cardiol. 2010; [online]. ahead print, PP.0-0)

Key words: Coronary artery disease; stents; myocardial revascularization; health policy.

Full texts in English - http://www.arquivosonline.com.br

Correspondência: George César Ximenes Meireles •

Rua Sena Madureira, 1265/102 - Vila Clementino - 04021-051 - São Paulo, SP - Brasil

E-mail: gcxm@cardiol.br, george.ximenes@terra.com.br

Artigo recebido em 03/10/08; revisado recebido em 08/06/09; aceito em 27/08/09. 


\section{Introdução}

O acometimento multiarterial é um dos principais preditores de prognóstico adverso em pacientes portadores de doença arterial coronariana ${ }^{1}$. Seu tratamento tem o objetivo de aliviar sintomas derivados da isquemia miocárdica, preservar a função ventricular esquerda e reduzir a incidência de eventos cardíacos adversos, promovendo maiores taxas de sobrevivência tardia naqueles submetidos a procedimento de revascularização miocárdica ${ }^{1,2}$. As melhorias na técnica de intervenção coronariana percutânea, configuração dos stents, guias, terapia antiplaquetária e stents farmacológicos tornaram-na uma opção de tratamento de pacientes com doença coronariana multiarterial, com taxas de mortalidade e infarto semelhantes à revascularização cirúrgica. Embora a intervenção coronariana percutânea esteja associada a risco significativamente maior para subsequentes revascularizações, a diferença entre as duas estratégias de tratamento vem diminuindo progressivamente: $34 \%$ nos estudos BARI/CABRI, 15\% no estudo ARTS e 5,5\% no recém-concluído estudo SYNTAX ${ }^{3-7}$.

As vantagens da intervenção coronariana percutânea em relação à cirurgia são o menor risco imediato de morbimortalidade cerebrovascular, pulmonar e renal, principalmente em pacientes idosos e em portadores de doença prévia destes sistemas; o tempo mais curto de convalescença e de internação hospitalar; o retorno precoce às atividades profissionais; e menores custos hospitalares e após a alta ${ }^{8}$. Dados recentes, incorporando os avanços tecnológicos no implante de stents e na cirurgia, demonstraram que os custos da hospitalização inicial e aos dois anos após o tratamento de pacientes com doença coronariana multiarterial com múltiplos stents convencionais versus CRM foram mais vantajosos para o grupo dos stents ( $27 \%$ de redução), a despeito de uma taxa maior de nova revascularização neste grupo ${ }^{9,10}$.

As intervenções coronarianas percutâneas em pacientes com doença multiarterial provenientes do SUS são realizados com stents convencionais e, em sua grande maioria, de forma escalonada (em mais de um procedimento), devido à limitação da quantidade de stent por procedimento, ocasionando um aumento desnecessário no número de procedimentos de implante de stent coronariano e, consequentemente, uma elevação de gastos públicos ${ }^{11}$. Os stents convencionais apresentam maiores taxas de nova revascularização da lesãoalvo ${ }^{12,13}$ em relação aos stents farmacológicos, prótese ainda não incorporada pelo SUS, contribuindo para a elevação do número de procedimentos necessários para o tratamento da reestenose do stent.

No Brasil, são escassos os estudos publicados sobre os aspectos econômicos relativos a procedimentos terapêuticos por intervenção coronariana percutânea ou por cirurgia de revascularização miocárdica para a doença arterial coronariana ${ }^{11,14-16}$.

O objetivo do presente estudo foi avaliar os valores remunerados pelo SUS para a obtenção da revascularização miocárdica percutânea completa em pacientes com doença multiarterial, relacionados ao número de procedimentos necessários e de stents implantados.

\section{Métodos}

Trata-se de um estudo prospectivo, realizado em hospital filantrópico de Guarulhos, São Paulo, gerenciado pela ordem religiosa Camiliana, em pacientes do SUS com doença multiarterial coronariana indicados eletivamente para a revascularização miocárdica percutânea.

Os pacientes faziam parte de protocolo de pesquisa comparativa do implante de stents coronarianos de cromo cobalto versus aço inoxidável em 200 pacientes com doença arterial coronariana multiarterial, com o objetivo de comparar a perda tardia e a taxa de reestenose aos 6 meses pósimplante entre os dois tipos de stents. Foram incluídos 141 pacientes, com doença coronariana multiarterial, submetidos à revascularização completa com sucesso pelo implante de stent convencional, sem limite de idade e sexo, e que tinham atingido os objetivos do protocolo inicial até a data do presente estudo.

O projeto de pesquisa foi analisado e aprovado pelo Comitê de Ética em Pesquisa do Instituto de Assistência Médica ao Servidor Público Estadual (IAMSPE) e os pacientes que participaram leram e assinaram o Termo de Consentimento Livre e Esclarecido.

Foram critérios de inclusão: ser elegível para a revascularização miocárdica percutânea ou cirúrgica; ser portador de angina estável ou síndrome coronariana aguda (angina instável ou infarto agudo do miocárdio) com múltiplas lesões obstrutivas coronarianas; ter lesões de novo potencialmente adequadas para o implante de stent, localizadas em artéria coronária principal ou ramo secundário; e fornecer consentimento por escrito, após ter sido informado sobre a natureza do estudo.

Foram critérios de exclusão: contraindicação para o uso de aspirina ou clopidrogel; fração de ejeção de ventrículo esquerdo inferior a 30\%; e doenças sistêmicas capazes de influenciar a sobrevida: insuficiência renal crônica, neoplasia maligna, doença do tecido conjuntivo, insuficiência hepática, insuficiência cardíaca, doenças trombogênicas e doenças cerebrovasculares.

\section{Definições}

Sucesso angiográfico: obtenção do percentual da estenose em diâmetro menor que $20 \%$ com fluxo TIMI 3, avaliado pela angiografia coronariana quantitativa on-line. Sucesso do procedimento: obtenção do sucesso angiográfico na ausência de eventos cardíacos maiores (morte, infarto ou cirurgia de emergência). Doença multiarterial coronariana: porcentual da estenose em diâmetro $\geq 70 \%$ em pelo menos duas artérias coronárias. Reestenose angiográfica: percentual da estenose em diâmetro > 50\% no local do implante de stent (dentro do stent ou até $5 \mathrm{~mm}$ proximal e distal à área do stent) na angiografia aos 6 meses pós-implante. Revascularização da lesão-alvo: cirurgia de revascularização miocárdica ou angioplastia por balão, envolvendo o segmento arterial do stent. Angioplastia para trombose subaguda de stent foi considerada revascularização da lesão-alvo. A coronariografia de controle foi realizada aos 6 meses do implante do último stent. A revascularização completa foi definida como o tratamento percutâneo de todas as lesões com porcentual 
de estenose $>70 \%$ em vasos com diãmetro $>2 \mathrm{~mm}$. Para análise dos custos, foram considerados os valores da Tabela $\mathrm{SIH} / \mathrm{SUS}$ de $\mathrm{R} \$ 2.263,77$ para o procedimento e $\mathrm{R} \$ 2.034,23$ por stent implantado.

Técnicas para aquisição de imagens: Para cada um dos pacientes, foram avaliadas as angiografias coronarianas para análise quantitativa pré e imediatamente pós-implante de cada stent e aos 6 meses pós-implante. Angiografia coronariana quantitativa: a análise quantitativa off-line do filme de cada paciente foi realizada por sistema automático de detecção de bordas do equipamento Philips do laboratório de hemodinâmica do hospital. Por meio desse sistema computadorizado de análise, foi determinada em cada uma das angiografias (pré, imediatamente pós-intervenção e no seguimento angiográfico): o diâmetro de referência interpolado do vaso-alvo; o diâmetro luminal mínimo e o percentual da estenose em diâmetro no local-alvo. Na angiografia pré-procedimento, foi também medida a extensão da lesão-alvo.

Avaliação da função ventricular esquerda: a função do ventrículo esquerdo foi expressa pela fração de ejeção global em percentual, mensurada na angiografia préprocedimento.

Os implantes dos stents foram realizados pelas técnicas atualmente preconizadas e de acordo com os critérios do SUS do uso de dupla prótese intraluminal arterial no mesmo procedimento, não excedendo $20 \%$ do total de angioplastias coronarianas realizadas.

Medicação concomitante: os pacientes foram medicados com aspirina 200 mg ao dia e clopidrogel 300 mg no dia anterior ao procedimento. Após a alta hospitalar, o clopidrogel 75 mg foi administrado por 30 dias e a aspirina indefinidamente.

Para análise estatística, os dados quantitativos foram apresentados como mediana, percentis 25 e 75, valores mínimos e máximos e variáveis qualitativas em porcentagem. Foi realizado o teste de qui-quadrado para associação de variáveis clínicas e angiográficas e reestenose e regressão logística dessas mesmas variáveis com a reestenose. Foi utilizado o programa de estatística Epi Info, versão 3.4.3 (disponível em: http://www.cdc.gov/epiinfo/).

\section{Resultados}

No período de julho de 2006 a dezembro de 2007 foram implantados 416 stents em 141 pacientes (mediana: 2; percentil 25: 2; percentil 75: 3; mínimo: 2 e máximo: 7). O número de vasos foi 356 pacientes (mediana: 2; percentil 25: 2; percentil 75: 3; mínimo: 1 e máximo: 6) e o número de lesões foi 416 (2,95 \pm 1,1 lesões/paciente). A proporção stents/vaso foi 1,17 .

As características clínicas dos pacientes estão listadas na Tabela 1. A idade média foi 59,7 $\pm 9,9$ anos, com predomínio do sexo masculino $(68,1 \%)$. Foi observado que $39 \%$ dos pacientes eram diabéticos e a função ventricular esquerda média era normal.

Para a obtenção da revascularização miocárdica completa pelo implante de stent coronariano foi necessário o escalonamento em até 4 procedimentos. O tempo médio entre a $1^{\underline{a}}$ e $2^{\underline{a}}, 2^{\underline{a}}$ e $3 \underline{a}$, e $3^{\underline{a}}$ e $4 \underline{a}$ angioplastias foi 45,8 $\pm 37,7$ dias, 55,4 \pm 55,3 dias e 33,5 $\pm 19,1$ dias, respectivamente.

A relação dos tempos dos procedimentos, número de pacientes e stents por procedimentos estão na Tabela 2 e a sua relação com os valores médios dos stents e procedimentos por paciente estão na Tabela 3. Podemos observar na Tabela 3 que em $73 \%$ dos pacientes (103) os implantes de stents foram realizados em mais de um tempo, o que ocasionou elevação dos valores na ordem de 26\%, 35,4\% e 30,3\% para 2, 3 e 4 tempos, respectivamente, caso fossem realizados em um único tempo.

A taxa de reestenose total, considerando as lesões tratadas, foi de $33,6 \%$ e quando comparados os diabéticos e não diabéticos foram observados $60 \%$ a mais de reestenose nos pacientes diabéticos (Tabela 4).

$\mathrm{Na}$ análise de regressão logística observou-se que foram fatores preditores independentes de reestenose o diabete melito e vasos $<2,75 \mathrm{~mm}$ (Tabela 5).

\section{Tabela 1 - Características basais dos pacientes}

\begin{tabular}{lc}
\hline Variáveis & $\mathrm{N}=141$ \\
\hline Idade (anos) & $59 \pm 9,9$ \\
\hline Sexo masculino & $95(68,1 \%)$ \\
\hline Tabagismo atual & $53(37,9 \%)$ \\
\hline Hipertensão arterial sistêmica & $107(75,9 \%)$ \\
\hline Hipercolesterolemia (colesterol total > 200 mg/dl) & $21(12,8 \%)$ \\
\hline Diabete melito (glicose <125 mg/dl) & $55(39 \%)$ \\
\hline Infarto do miocárdio prévio & $26(18,4 \%)$ \\
\hline Cirurgia de revascularização do miocárdio prévia & $21(12,8 \%)$ \\
\hline História familiar & $88(62,4 \%)$ \\
\hline Angina estável & $61(43,3 \%)$ \\
\hline Síndrome coronariana aguda sem supra de ST & $55(39 \%)$ \\
\hline Síndrome coronariana aguda com supra de ST & $25(17,7 \%)$ \\
\hline Fração de ejeção do ventrículo esquerdo & $64,2 \pm 14,7$ \\
\hline
\end{tabular}

Tabela 2 - Escalonamento dos implantes de stents

\begin{tabular}{lccccc}
\hline \multirow{2}{*}{ Número stents } & \multicolumn{4}{c}{$\begin{array}{c}\text { Número de pacientes por tempo de } \\
\text { implante de stents }\end{array}$} & $\begin{array}{c}\text { Total } \\
\text { pacientes }\end{array}$ \\
\cline { 2 - 5 } & 1 & 2 & 3 & 4 & \\
\hline 2 stents & 35 & 27 & 0 & 0 & 62 \\
\hline 3 stents & 3 & 32 & 10 & 0 & 45 \\
\hline 4 stents & 0 & 10 & 10 & 0 & 20 \\
\hline 5 stents & 0 & 0 & 6 & 2 & 8 \\
\hline 6 stents & 0 & 1 & 4 & 0 & 5 \\
\hline 7 stents & 0 & 0 & 0 & 1 & 1 \\
\hline Total & 38 & 70 & 30 & 3 & 141 \\
\hline
\end{tabular}


Tabela 3 - Custos de acordo com o escalonamento e número de stents

\begin{tabular}{lccccc}
\hline \multirow{2}{*}{$\begin{array}{l}\text { Número } \\
\text { stents }\end{array}$} & \multicolumn{4}{c}{ Custos (R\$) por paciente e por tempos de } & Total \\
\cline { 2 - 6 } & 1 & 2 & 3 & 4 & pacientes \\
\hline 2 stents & $6.332,20$ & $8.596,00$ & - & - & 62 \\
\hline 3 stents & $8.366,46$ & $10.630,20$ & $12.894,00$ & - & 45 \\
\hline 4 stents & - & $12.664,50$ & $14.928,23$ & - & 20 \\
\hline 5 stents & - & - & $16.962,46$ & $19.226,23$ & 8 \\
\hline 6 stents & - & $16.732,92$ & $18.999,93$ & - & 5 \\
\hline 7 stents & - & - & - & $23.294,69$ & 1 \\
\hline $\begin{array}{l}\text { Total } \\
\text { pacientes }\end{array}$ & 38 & 70 & 30 & 3 & 141 \\
\hline
\end{tabular}

Tabela 4 - Associação entre diabete e reestenose

\begin{tabular}{lccc}
\hline & Sem reestenose & Com reestenose & Total \\
\hline Sem diabete & $181(63 \%)$ & $68(27 \%)$ & 249 \\
\hline Com diabete & $92(56,8 \%)$ & $70(43,2 \%)$ & 162 \\
\hline Total & $273(66,4 \%)$ & $138(33,6 \%)$ & 411 \\
\hline
\end{tabular}

$p=0,001$.

Tabela 5 - Fatores associados com a reestenose na análise por regressão logística

\begin{tabular}{lcc}
\hline Variável & $\begin{array}{c}\text { Odds Ratio (intervalo de } \\
\text { confiança 95\%) }\end{array}$ & $\mathbf{p}$ \\
\hline Diabete & $1,81(1,2-2,7)$ & 0,0045 \\
\hline Vaso Pequeno $(<2,75 \mathrm{~mm})$ & $0,52(0,37-0,72)$ & 0,0001 \\
\hline Extensão $\geq 20 \mathrm{~mm}$ & $1,1(0,69-1,75)$ & 0,68 \\
\hline
\end{tabular}

\section{Discussão}

O presente estudo analisa os valores remunerados pelo SUS para a realização da revascularização completa pelo implante de stents coronarianos em pacientes com doença coronariana multiarterial atendidos em hospital terciário de referência para realização de intervenções coronarianas percutâneas do SUS em Guarulhos, segunda maior cidade em população do estado de São Paulo, com aproximadamente um milhão e duzentos mil habitantes.

Os estudos de custos de procedimentos médicos levam a resultados que não podem ser generalizados para outros países nem para outras regiões de um mesmo país, pois dependem da estrutura econômica na qual o cuidado à saúde está inserido. O pagamento das intervenções coronarianas percutâneas realizado pelo SUS para a rede credenciada é realizado na forma de pacote fechado, que inclui o valor do procedimento e do stent, limitado a dois stents em até $20 \%$ dos procedimentos e não levam em conta a duração da internação ou a ocorrência de complicações do procedimento tratadas pelo implante de stent adicional. No presente estudo, foi necessário o implante de três stents por procedimento em 4 pacientes, ultrapassando o limite estabelecido.

Os estudos publicados no Brasil são comparativos dos custos da revascularização miocárdica por meio da intervenção coronariana percutânea ou pela cirurgia de revascularização miocárdica e são relativos a estimativas de custos hospitalares e de materiais, não considerando o valor do pacote pago pelo SUS $11,14,15$.

A intervenção percutânea em pacientes multiarteriais apresenta, em relação à cirurgia, a possibilidade do procedimento ser escalonado na dependência da apresentação clínica, função renal, complexidade das lesões, função ventricular e acesso vascular ${ }^{17}$. Em estudo, para avaliação dos custos do implante programado de múltiplos stents em mais de uma intervenção percutânea em pacientes do SUS com doença coronariana multiarterial, observou-se concordância em $44 \%$ com o escalonamento considerado ideal pela equipe de cardiologia intervencionista e discordância em $56 \%$, o que gerou custos adicionais dos valores referentes aos procedimentos no valor de $\mathrm{R} \$ 131.298,70$. Os critérios utilizados para o escalonamento do implante de stents pela equipe de cardiologia intervencionista foram: dificuldade de acesso, anatomia coronariana desfavorável (ex.: intensa angulação proximal), lesões complexas (tipo C), insuficiência cardíaca ou renal e síndrome coronariana aguda ${ }^{11}$.

Podemos observar no presente estudo que, em 73,1\% dos pacientes com doença coronariana multiarterial que necessitaram de implante de múltiplos stents, o procedimento foi realizado de forma escalonada. No estudo SYNTAX, recentemente concluído, comparativo das técnicas de intervenção coronariana percutânea com stent farmacológico e cirurgia de revascularização miocárdica, a maioria dos implantes de stents no grupo das intervenções coronarianas percutâneas foi realizada em um único procedimento, escalonado em $14,1 \%$ dos pacientes, cuja média de implante foi de 4,6 stents por paciente ${ }^{7}$.

Como o preço do stent é fixo, fica muito claro que o aumento do número de procedimentos é o que ocasiona a elevação dos valores, além de maior sofrimento e risco de complicações para os pacientes. Os responsáveis pela tomada de decisões do SUS deverão buscar soluções no sentido de que a decisão pelo número de intervenções coronarianas necessárias para a obtenção da revascularização completa é de competência do médico cardiologista intervencionista, em conjunto com o cardiologista clínico ${ }^{11}$.

Outra questão importante a ser abordada é o tipo de stent utilizado. Apesar da conhecida superioridade dos stents farmacológicos em relação aos stents convencionais em reduzir a necessidade de novas revascularizações da lesãoalvo, não foram utilizados tais stents neste estudo devido a sua não incorporação pelo SUS. Quando comparados a utilização dos stents convencionais e a cirurgia de revascularização miocárdica para tratamento da doença coronariana multiarterial, observou-se em metanálise recente, incluindo 23 ensaios randomizados com mais de 10.000 pacientes, que ao final de 5 anos de seguimento clínico não foi observada diferença significativa na mortalidade entre as duas terapêuticas, mas a cirúrgica forneceu maior durabilidade tardia da revascularização, com taxas menores de novos 
procedimentos $^{18}$. Quando a intervenção coronariana em multiarteriais foi realizada com stents farmacológicos e comparada aos resultados cirúrgicos, ocorreu uma grande redução da taxa de revascularização e, dependendo da complexidade das lesões, ainda mais favorável para a cirurgia ${ }^{7}$.

Dentre as variáveis clínicas, o diabete melito é reconhecido como a única variável com poder independente para a ocorrência de maiores taxas de reestenose coronariana. O sinergismo potencial da associação com o tratamento de estenoses mais longas localizadas em vasos de menor calibre eleva progressivamente os riscos para sua ocorrência ${ }^{19-21}$. No presente estudo observou-se taxa total de reestenose de 33,6\% e que o diabete melito e vasos com diâmetros $<2,75 \mathrm{~mm}$ foram preditores independentes de reestenose (Tabelas 4 e 5). $\mathrm{O}$ advento dos stents farmacológicos promoveu significativo impacto na redução das taxas de reestenose e da necessidade de repetir procedimentos de revascularização do miocárdio em pacientes diabéticos ${ }^{22}$

Os valores da tabela SUS para stents convencionais estão muito acima do custo real para as instituições de saúde. Então, com uma política de preços justos para esses stents, os recursos economizados poderiam ser aplicados no uso de stents farmacológicos, limitados aos pacientes com maior probabilidade de reestenose (vasos pequenos, lesões muito longas, reestenose intrastent e em pacientes com diabete melito). A nossa experiência em uma instituição pública (IAMSPE), que incorporou o uso de stents farmacológicos para tratamento da doença arterial coronariana obstrutiva, permite

\section{Referências}

1. Alderman EL, Bourassa MG, Cohen LS, Davis KB, Kaiser GG, Killipi T, et al. Ten-year follow-up of survival and myocardial infarction in the randomized Coronary Artery Surgery Study. Circulation. 1990; 82: 1629-46.

2. King SB 3rd, Smith SC Jr, Hirshfeld JW Jr, Jacobs AK, Morrison DA, Williams DO. 2007 Focused Update of the ACC/AHA/SCAI 2005 Guideline Update for Percutaneous Coronary Intervention: a report of the American College of Cardiology/American Heart Association Task Force on Practice Guidelines: 2007 Writing Group to Review New Evidence and Update the ACC/AHA/SCAI 2005 Guideline Update for Percutaneous Coronary Intervention, Writing on Behalf of the 2005 Writing Committee. Circulation. 2008; 117: 261-95.

3. First-year results of CABRI (Coronary Angioplasty versus Bypass Revascularization Investigation). CABRI Trial Participants. Lancet. 1995; 346: $1170-84$

4. Comparison of coronary bypass surgery with angioplasty in patient with multivessel disease. The Bypass Angioplasty Revascularization Investigation (BARI) Investigators. N Engl J Med. 1996; 335: 217-25.

5. Serruys PW, Unger F, Sousa JE, Jatene A, Bonnier HG, Schomberger JP, et al. Comparison of coronary-artery bypass surgery and stenting for the treatment of multivessel disease. N Engl J Med. 2001; 344: 1117-24.

6. Hoffman SN, Tenbrok JA, Wolf MP, Pauker SG, Salem DN, Wong JB. A metaanalysis of randomized controlled trials comparing coronary artery bypass graft with percutaneous transluminal coronary angioplasty: one- to eight-year outcomes. J Am Coll Cardiol. 2003; 41: 1293-304.

7. Rastan AJ, Boudriot E, Falk V, Kappetein AP, Borger MA, Serruys PW, Mohr FW, et al. Frequency and pattern of the novo three vessel and left main coronary artery disease; insights from single center enrolment in the SYNTAX study. Eur J Cardiothorac Surg. 2008; 34 (2): 376-82.

8. Zago AC, Sousa AGMR, Sousa JE. Evolução da intervenção percutânea para estimar o uso do stent farmacológico em aproximadamente $25 \%$ das intervenções coronarianas. Os provedores de serviços de saúde devem utilizar como referência o Consenso de Especialistas (SBC/SBHCl) sobre o Uso de Stents Farmacológicos e as recomendações da recém-publicada Diretrizes da SBCHI - Intervenções Coronarianas Percutâneas e Métodos Diagnósticos em Cardiologia Intervencionista ${ }^{23,24}$.

\section{Conclusão}

A revascularização percutânea completa em pacientes do SUS com doença coronaria multiarterial, realizada em sua grande maioria de forma escalonada, ocasiona considerável elevação de gastos públicos devido ao aumento do número de procedimentos.

\section{Potencial Conflito de Interesses}

Declaro não haver conflito de interesses pertinentes.

\section{Fontes de Financiamento}

$\mathrm{O}$ presente estudo não teve fontes de financiamento externas.

\section{Vinculação Acadêmica}

Não há vinculação deste estudo a programas de pósgraduação. o tratamento da doença coronária multiarterial. Arq Bras Cardiol. 2002; 78 : $250-60$

9. Reynolds MR, Neil N, Ho KK, Berezin R, Cosgrove RS, Larger RA, et al Clinical and economic outcomes of multivessel coronary stenting compared with bypass surgery: a single-center US experience. Am Heart J. 2003; 145: 334-42.

10. Nagle OS, Smith AW. Review of recent US cost estimates of revascularization Am J Manag Care. 2004; 10: 370-6.

11. Pedroso AP, Meireles GCX, Abreu Filho LM, Forte AAC, Sumita MK, Favarato D, et al. Avaliação do custo do implante programado de múltiplos stents em mais de uma intervenção percutânea em pacientes com doença coronária multiarterial. Rev Bras Cardiol Invas. 2007; 15: 249-54.

12. Babapulle MN, Joseph L, Bélisle P, Brophy JM, Eisenberg MJ. A hierarchica Bayesian meta-analysis of randomised clinical trials of drug-eluting stents. Lancet. 2004; 364: 583-91

13. Stettler C, Wandel S, Allemann S, Kastrati A, Morice MC, Schömig A, et al Outcomes associated with drug-eluting and bare-metal stents: a collaborative network meta-analysis. Lancet. 2007; 370: 937-48.

14. Favarato D, Hueb W, Gersh BJ, Soares PR, Cesar LAM, Da Luz PL, et al. Relative cost comparison of treatment for coronary artery disease: the first year followup of MASS II study. Circulation. 2003; 108 (Suppl II): II21-II23.

15. Almeida RMS. Revascularização do miocárdio - estudo comparativo do custo da cirugia convencional e da angioplastia transluminal percutânea. Rev Bras Cir Cardiovasc. 2005; 20: 142-8.

16. Hadda N, Bittar E, Marchi AF, Kantorowitz CSV, Ayoub AC, Fonseca ML, et al. Custos hospitalares da cirurgia de revascularização do miocárdio em pacientes coronarianos eletivos. Arq Bras Cardiol. 2007; 88: 418-23.

17. Zimarino M, Calafiore AM, De Catarina R. Complete myocardial 
Meireles e cols.

Análise dos valores SUS em multiarteriais

revascularization: between myth and reality. Eur Heart J. 2005; 26: 1824-30.

18. Bravata DM, Gienger AL, McDonald KM, Sundaram V, Perez MV, Varghese $\mathrm{R}$, et al. Systematic review: the comparative effectiveness of percutaneous coronary interventions and coronary artery bypass graft surgery. Ann Intern Med. 2007; 147: 703-16.

19. Kastrati A, Schömig A, Elezi S, Schühlen H, Dirschinger J, Hadamitzky M, et al. Predictive factors of restenosis after coronary stent placement. J Am Coll Cardiol. 1997; 30: 1428-36.

20. Cutlip DE, Chauhan MS, Baim DS, Ho KK, Popma JJ, Carrozza JP, et al. Clinical restenosis after coronary stenting: perspectives from multicenter clinical trials. J Am Coll Cardiol. 2002; 40: 2082-9.

21. Mauri L, Orav EJ, Kuntz RE. Late loss in lumen diameter and binary restenosis for drug-eluting stent comparison. Circulation. 2005; 111: 3435-42.

22. Boyden TF, Nallamothu BK, Moscucci M, Chan PS, Grossman PM, Tsai TT, et al. Meta-analysis of randomized trials of drug-eluting stents versus bare metal stents in patients with diabetes mellitus. Am J Cardiol. 2007; 99: 1399-402.

23. Sociedade Brasileira de Cardiologia. Sociedade Brasileira de Hemodinâmica e Cardiologia. Intervenção coronária percutânea e métodos adjuntos diagnósticos em cardiologia intervencionista. Rev Bras Cardiol Invas. 2008; 16 (supl.2): 9-88.

24. Sociedade Brasileira de Cardiologia. Consenso de especialistas ( $\mathrm{SBC} / \mathrm{SBHCl}$ ) sobre o uso de stents farmacológicos: recomendações da Sociedade Brasileira de Hemodinâmica e Cardiologia Intervencionista ao Sistema Único de Saúde. Arq Bras Cardiol. 2006; 84 (4): e162-e167. 